\title{
One Thing After Another: Why the Passage of Time is Not an Illusion
}

(forthcoming in The Illusions of Time: Philosophical and Psychological Essays on Timing and Time Perception, Adrian Bardon, Valtteri Arstila, Sean Power \& Argiro Vatakis (eds.), Palgrave Macmillan)

\begin{abstract}
:
Does time seem to us to pass, even though it doesn't, really? Many philosophers think the answer is 'Yes' - at least when 'time's (really) passing' is understood in a particular way. They take time's passing to be a process by which each time in turn acquires a special status, such as the status of being the only time that exists, or being the only time that is present (where that means more than just being simultaneous with oneself). This chapter suggests that on the contrary, all we perceive is temporal succession, one thing after another, a notion to which modern physics is not inhospitable. The contents of perception are best described in terms of 'before' and 'after', rather than 'past', 'present, and 'future'.
\end{abstract}

Is the passage of time an illusion? This has meant many different things to different people. As rightly noted by Gruber et al. (2018), both 'passage of time' and 'illusion' invite a multitude of interpretations. Moreover, it's a question that cries out for interdisciplinary efforts (such as this volume), since numerous disciplines - including physics, cognitive science, developmental psychology, evolutionary biology and philosophy - are potentially relevant. It's also a very timely question (no pun intended). For example, time has recently taken center stage at the frontiers of physics. Physicists working towards a theory of quantum gravity have had to re-examine some very basic assumptions about time, such as whether it exists, and if it does, whether it is fundamental or in some sense emergent (Callender, 2010).

The title of this chapter might lead one to expect a theory of what temporal passage is, and an argument for the view that it's not an illusion. In fact, my aims are a bit more narrowly circumscribed. First, I'll describe what 'passage' stands for in current 
philosophical usage. For there to be an illusion of passage, one would have to answer 'Yes' and 'No', respectively, to the following two questions: '(a) does time seem to pass?', and '(b) does it pass?'. Assuming for present purposes that the answer to (b) is indeed 'No', I'll suggest that the answer to (a) is also 'No'. The upshot will be that there is no illusion of passage: all we perceive is one thing after another.

\section{1 'The passage of time'}

The phrase 'the passage of time' (or 'the passing of time', or 'temporal passage', or 'temporal flow') has a particular meaning in philosophical debates about the nature of time. Passage is a (putative) feature that time is supposed to be able to have or lack, while in any case existing. That is, we're not here concerned with the possibility that time may not exist, nor with the possibility that it may not be fundamental. (So this is not directly about those basic questions arising from within current research in physics.) Rather, the question is whether or not time, a real feature of the world, itself has the feature of being such that it passes. This question may well sound a little strange at first. Ordinary usage may not allow much leeway between time existing and time passing. But philosophical reflection has produced a distinction between two ways that time could in principle be, namely dynamic (such that it passes) or non-dynamic. To illustrate, consider a few ideas for what time's passing might consist in, according to different dynamic views of time: time would pass in this sense if only one time was (ever) real, so that times came into and went out of existence constantly; or, if only the past and the present were real, so that reality as a whole grew constantly; or, if future events constantly became less and less future, until they became present and then more and more past. All these views have in common that they take the metaphors about time being like a river, exhibiting some kind 
of sui generis movement of its own, very seriously. On these views, time is not much like space, at least not in this respect. Space doesn't pass, after all.

All these dynamic views (versions of the A-theory) are opposed by the nondynamic, or block universe view (also called the B-theory). The block universe view denies that time does anything like passing in this sense. At least in this respect, time is no different from space.

Though it's the product of philosophical reflection, the distinction between dynamic and non-dynamic views of time is very intuitive at root. After all, the metaphors that drive the distinction are commonplace in many languages (though the details reveal many fascinating differences as well, see section 4). We commonly speak as if we were moving through time, or as if time and events were moving past us. Is time really like this, or does it lack the dynamic features the metaphors point to? It's not surprising that something like this question has left traces throughout the history of philosophy. The British idealist philosopher J. M. E. McTaggart brought it into sharp focus (arguing that for time to exist it would have to be dynamic, but it can't be, so it doesn't exist), but its history stretches back at least to Heraclitus and Parmenides.

Its history is also intertwined with the history of time in physics. The block universe view has often been associated with time in relativity theory. One not so good reason for this is the four dimensional nature of relativistic spacetimes. On a spacetime diagram, past present and future are seemingly already there. There is no dynamism, just the block universe. This isn't a good reason to associate the B-theory with time in relativity theory, mainly because there are spacetime formulations of non-relativistic physics too. But there are other reasons. In particular, all the dynamic views seem to rely 
on there being a 'now' that is the same everywhere in space. But relativity teaches us that there is no objective notion of distant simultaneity, of the same time at different spatial locations. What's simultaneous with what depends on one's state of motion. So this makes trouble for any dynamic view that says that what's dynamic is some global present, ever moving on, or constituting the edge of what exists, or the only time that exists. By elimination, the block universe view emerges looking vindicated. Philosophers and physicists alike have expressed the sense that relativity theory shows time to be very much unlike the metaphors suggest. Hermann Weyl famously remarked that ' $[\mathrm{t}] \mathrm{he}$ objective world simply is, it does not happen. Only to the gaze of my consciousness, crawling upwards along the life line of my body, does a section of this world come to life as a fleeting image in space which continuously changes in time' (Weyl, 1949).

Admittedly, this almost sounds like a dynamic view of time again, but only almost: time itself is non-dynamic; 'only to the gaze of my consciousness' does temporal reality present itself as dynamic. Similarly, Arthur Eddington said that '[e]vents do not happen; they are just there and we come across them' (Eddington, 1920). Albert Einstein himself went so far as to claim spiritual significance for this view of time. Shortly after the death of his friend Michele Besso, he wrote: 'Now he has also gone ahead of me a little in departing from this peculiar world. This means nothing. For us believing physicists, the division between past, present and future has only the significance of a stubbornly persistent illusion.' (Einstein, 1972)

\section{2 'Illusion'}

This brings us to the term 'illusion'. Roughly, I'll take this to mean a mismatch between how things seem, and how they are. In this case, the mismatch would be between time's 
being non-dynamic and yet seeming dynamic. Thus, the question at hand is whether two things are the case: time doesn't pass (in the sense of the A versus B debate), and yet it seems to (in that same sense). (From now on 'passage' will denote passage in the sense of the A versus B debate.) Let's call the corresponding questions '(a) does time seem to pass?' and '(b) does time pass?'. And let's assume for present purposes that time doesn't pass, and concentrate on (a): does it seem to? That is, do we have passage phenomenology?

Is passage something one should even expect to show up in experience? Some are skeptical about this. Suppose that the passage of time turns out to be the 'birthing' of new elements of a causal set, i.e. discrete spacetime points. This is a suggestion recently made by some of the physicists working on causal set theory, which is intended to be a stepping stone towards a theory of quantum gravity. Isn't the growth of causal sets the last thing one should expect ordinary experience to be responsive to? Since when does our phenomenology track the most fundamental layer of reality as revealed by our best physical theories? How likely is it that when I glance at my watch, I somehow intimate the stochastic growth process constituting spacetime itself? On the other hand, those physicists writing about this seem to be motivated precisely by the link to experience. For example, Rafael Sorkin writes: “[S]equential growth $[\ldots]$ provides an objective correlate of our subjective perception of "time passing" in the unceasing cascade of birth-events that build up the causal set [...].” (Sorkin, 2007). More generally, as the Weyl and Einstein quotes already suggest, it's somewhat natural to think that a non-dynamic temporal reality is at odds with how things seem. There's something striking about the claim that time doesn't pass, and presumably the reason is that it seems to. 
Forget passage for a moment and just consider our experience of time as such. That is, just consider our experiences as of temporal duration, of events as occurring in a particular order, and as following on from or succeeding one another. ('As of $\mathrm{x}$ ' is meant to leave it open whether the experience is veridical or illusory; that is, one can have an experience as of $\mathrm{x}$ without there being any $\mathrm{x}$.) In order to understand the question at hand, namely '(a) does time seem to pass?', one has to keep in mind that both sides to this dispute can and typically do allow that we have temporal experiences. Whether we have experiences as of time passing or not, we definitely have experiences as of succession, order, and duration. However, those who answer 'Yes' to (a) think that we only have experiences as of succession, order, and duration, by having experiences as of time passing, or because we have experiences as of time passing. For them, it's the impression of dynamicity that enables or underlies the impression of succession, order, and duration. Some speak of 'animated change' in this connection (Paul, 2010). So the question is, do we experience temporal features of the world only by experiencing time's passing? Does our temporal phenomenology involve passage phenomenology? Or just impressions of succession, order, and duration?

So far, I've talked of 'experiences', 'impressions' and 'phenomenology'. But when it comes to time, there is one rough distinction that it's useful to make at the outset. This is the distinction between our perceptual experiences (or perceptions) and experiences in a broader sense (let's call these EXPERIENCES). Our (perceptual) experiences of time unfold over small time scales. They include seeing the second hand move around the clock, hearing a succession of notes, or feeling a raindrop run down one's neck. Our EXPERIENCES unfold over a wide range of time scales longer, all of 
them longer than the time scales relevant to (perceptual) temporal experiences. The following are all examples of temporal EXPERIENCES: noticing that the hour hand has moved around the clock, reflecting on how a city has changed over the years, and feeling as if time somehow passes more quickly as one gets older. These EXPERIENCES are quite varied, and it would be no easy to task to say more precisely what distinguishes them from (perceptual) experiences, or how the two categories are related. But (perceptual) experiences of time are a good starting point. Does seeing the second hand move involve seeing the passing of time?

3 Why we don't perceive time's passing

With this intended meaning of (a) firmly in mind, it would be reasonable to worry that it won't be easy to decide on an answer. But here is a consideration that (indirectly) speaks in favor of 'No'.

Consider what happens when one answers 'Yes', and when one combines that with the claim that time does not pass (answering 'No' to (b)). This is the view that the passage of time is an illusion. It lends itself to a methodology whereby one looks to insights in cognitive science work on perceptual illusions, and sees whether they can be applied here. One influential proposal of this kind is due to philosopher Laurie Paul. Paul asks us to consider cases of illusory perception of motion, such as films, time-lapse photography, or old-fashioned flipbooks. Another central example for Paul is (what in the philosophical literature has become known as) the 'color phi' experiment. If a subject is presented with appropriately spaced and timed flashes of differently colored dots on opposite sides of a screen, it can seem to them as though a single dot is continuously moving back and forth and changing color mid-trajectory. So here we have static inputs 
of the form 'red flash left' and 'green flash right', leading to dynamic outputs, namely the impression of a single dot moving back and forth, changing color. The idea is that something analogous might be underlying our sense of passage. Although the inputs are 'static', passage-less facts, like 'object $\mathrm{O}$ has property $\mathrm{P} 1$ at $\mathrm{t} 1$ ' and 'object $\mathrm{O}$ has property $\mathrm{P} 2$ at $\mathrm{t} 2$ ', what we actually perceive is an animated change in $\mathrm{O}$, in which temporal passage is the key ingredient. In a nutshell, Paul's proposal likens time in the block universe to something like a film, giving rise to illusory passage phenomenology.

I think this proposal fails in an interesting way. Recall that all parties to the debate agree that there is change and succession in the block universe, and that our temporal phenomenology in a block universe includes experiences as of change and succession. The question is how to explain the additional illusory element of animation underlying this phenomenology. But on closer inspection, the proposed explanation suggests that there wasn't this additional element in the first place. Consider again the analogy between the 'static' block universe facts, and the static inputs in the color phi experiment. In the latter case, the brain's limited powers of discrimination lead to the gaps between images not being perceived. The problem is that facts about what happens when in the block universe aren't 'static' in the same sense: these facts are not themselves in time. Moreover, there need not be any gaps in the block universe. Whether or not time is continuous is orthogonal to the A versus B debate. But in order for the analogy to work, we have to pretend that there is discreteness in the block universe, or rather a 'gappiness' of the kind found in the color phi phenomenon.

Suppose there was. Then plausibly, what we would have explained would be an illusion of continuity - of continuous motion and persistence. In a gappy block universe, 
the brain produces experiences as of non-gappiness. But if that's all that needed explaining, then there wasn't an additional illusion of 'animation' (A-theoretic passage) to our temporal phenomenology in the first place. Only experiences as of continuous motion, of order, duration and succession.

Note that none of this bears on whether our temporal phenomenology is in some other respect illusory. In particular, what I've argued is compatible with our perception of motion and other change involving an illusion of continuity (though it's also compatible with this not being the case). ${ }^{1}$ It's worth noting this, because some of the same cognitive science work that Paul draws on has been presented as evidence for claims of that kind. Consider, for example, the position defended by Gruber et al. They distinguish between a upper-level and a lower-level 'flow of time', by which they mean two distinct aspects of our temporal phenomenology. The upper-level component they describe as a false cognition, and as having (what I'm calling) A-theoretic content: 'There is an upper-level component, the past/present/future, which entails a moving present' (Gruber et al., 2018, 133). The lower-level component they describe as 'the experience of events flowing or happening, such as occurs when a volcano erupts' (Gruber et al., 2018, 133); '[i]t involves visual, auditory, olfactory, and other sensory modalities' (Gruber et al., 2015, 9). Since the volcano example is presumably generic, by the "lower-level component of the flow of time', they just mean our perception of change, rather than a perception of passage. (Recall that all sides to the dispute I'm focusing on typically agree that our temporal phenomenology involves perceptions of change, but that only one side takes these to involve passage phenomenology.) Now, Gruber et al. argue that the lower-level component is illusory, because it is the "perceptual completion that provides continuity to 
discrete observation' (Gruber et al., 2018, 134). They go on to cite the phi phenomenon as part of the evidence for this claim, and they also make a connection with Paul's work: 'These views as to the illusory nature of motion are in good agreement with philosophical views. Paul (2010) argued that, in general, the flow associated with motion (a change of location of a persisting object) is merely an effect of the brain' (Gruber et al., 2018, 135). The point to note is that none of my arguments bear on whether we should think that our perception of change involves an illusion of continuity. My topic is whether temporal phenomenology is illusory in a different respect, namely in that it involves passage phenomenology. Incidentally, this is also the topic of Paul's proposal (as well as that of Christoph Hoerl's and Simon Prosser's writings in this area, who are also cited in this context by Gruber et al. $(2015,10,12 ; 2018,128)$. And this is so in spite of the fact that, as we've seen, Paul makes use of a lot of the same experimental results as Gruber et al., and in spite of the fact that she builds her proposal on an analogy with illusory perceptions of continuous motion.

We've seen one (indirect) reason to think we don't have passage phenomenology. Here are some more. Recall that in this discussion 'passage' means an updating of which time is 'special', for example which time is the one time that exists, or the latest time that exists, or the one time that is objectively present. Thus, in order for it to seem like time passes, one time and its contents should seem 'special'. Does it? Suppose it's Tuesday today. Do your Tuesday experiences have a special phenomenal character? Suppose you spent all of today, and all of yesterday, in a completely red room (Skow, 2015, 203), meditating while looking at the red walls. And suppose you're able to produce perfectly accurate descriptions of your phenomenology. Will your descriptions of your experiences 
on Monday and on Tuesday differ in some way? Do the Tuesday experiences feel somehow more vivid or more lustrous or in some other way 'special'? Presumably not: instead, the descriptions will be exactly the same. Presentness makes no difference to how things look or feel or sound.

Of course, perceiving something is different from remembering or anticipating it. So, in that sense, there is a connection between presentness and experience. At each time, one perceives only some things and not others. But this is a kind of 'specialness' that is compatible with the block universe: each time is 'present' and therefore 'special' at itself, just like each spatial location is 'here' at itself. No illusion there.

What, then, do we perceive, if not the passing of time? We perceive temporal succession, temporal order, and temporal duration. Our temporal phenomenology includes all of that. We are perceptually aware of changes and of persistence (things existing over time). These perceptual experiences present the world in a way that is perfectly compatible with the block universe view. If this is right, then perceptual contents are not best described using notions such as 'past, 'present' or 'future'. When we perceive a succession of events, we don't experience some things as present while experiencing other things as past (and perhaps still other things as future). Rather, perceptual contents are best described using notions like 'before' and 'after'. When we perceive a succession of events, we simply experience one event as happening after the other.

This way of thinking about temporal experiences may enjoy additional support from another direction. In particular, it may be well aligned with certain independently plausible positions in a different philosophical debate about the nature of temporal 
experience. This other debate has a long and even more illustrious history than the one we're concerned with, with key historical protagonists including William James and Edmund Husserl. There is some disagreement about how best to set it up (see e.g. Phillips, 2014). Descriptions of what's at stake range from 'how is temporal experience possible?', to 'how does the temporal structure of experience relate to the temporal structure of what is experienced?', to simply 'what is the nature and content of temporal experience?'. Interestingly, some approaches to these questions center on the idea that an experience of something temporally extended is itself temporally extended. The experience itself takes time to unfold - in fact it takes as much time as the process that it is an experience of. Arguably, this makes it easier to understand the nature of temporal experience. We no longer have to ask how it is that multiple individual experiences, each succeeding the other, can add up to an experience of succession. Instead, we recognize that the fundamental experiential unit is itself temporally extended, and use this to explain how there can be an experience of a temporally extended content. For present purposes, what's interesting about these approaches is that they may be particularly well aligned with the view that we don't perceive time as passing. Christoph Hoerl argues that if the experience takes as long as what's experienced, then 'there is nothing that falls within the experience to which the notion 'a short while ago' could apply, because there is nothing experienced as having happened at a time that pre-dates the onset of the experience' (Hoerl, 2009). Temporal perceptual contents are then not temporally viewpointed, in the way they would be if we experienced the passing of time.

4 Do we think time passes?

In the growing philosophical literature on negative answers to (a) (does time seem to 
pass?), a common thread is that if you answer 'No', you have a lot of explaining to do. In particular, you owe an explanation for why people were ever attracted to the view that time passes (why people tend to answer 'Yes' to (b)), and you owe an explanation for why people tend to think they experience time as passing (why people tend to answer 'Yes' to (a)).

Kristie Miller and co-authors explore different possible relations between these explanations (Miller et al, forthcoming). Perhaps one and the same mechanism explains both (our view that time passes and our view that we experience time as passing). Or maybe something makes us think time passes, and we sub-personally infer from this belief that we experience time's passing. Either way, the proposed explanation of why we think time passes is roughly this. First, we conceptualise time as having a direction, due to the many temporally asymmetric phenomena are all around and within us. This is reflected in the fact that many natural languages are direction-friendly, in the sense that they make it easy to express a conception of time as directed. Moreover, our lives are temporally ordered series of temporal perspectives, each of which includes mental representations of decisions at earlier times and deliberations about decisions at later times (Ismael 2010, 2012). To allow us to express how things are from each temporal perspective, languages need to be minimally passage-friendly: they need to include tense, or at least deictic adverbials like 'tomorrow' or 'yesterday'. Some are even substantially passage-friendly, in that they include moving time ('Christmas is approaching') or moving ego metaphors ('he's nearing his 70th birthday'). There is much evidence that language influences the way we think. So these facts about natural languages can in turn explain why we tend to think time passes. 
This is a fascinating explanation. Nonetheless, in what remains I'll suggest that one can take on board what's most convincing in it without assuming so uniform an explanandum as that 'we' tend to think time passes. This assumption is, as the authors note, widespread in this area of philosophy. But there are good reasons not to make it.

To begin with, as their article shows indirectly, there is fascinating variation in how different languages treat time. Not all languages are 'substantially passage-friendly'. Yet, it's precisely these moving time and moving ego metaphors that drive the A versus B debate. A-theories posit temporal passage in a way that is supposed to make these metaphors literal; the block universe view (B-theory) says time doesn't pass, in that same sense. Meanwhile, the universal 'minimal passage-friendliness', which characterizes all languages, barely goes beyond 'direction-friendliness'. The former allows one to express degrees of pastness or futurity with respect to the present utterance (whether via tense or not); the latter 'make it easy to distinguish what has been the case from what is the case, and what will be the case'. This too is revealing, because direction-friendliness can't by itself dispose one towards an A-theoretic conception (on which time passes), for the simple reason that the directionality issue is different from the passage issue. It's perfectly possibly to think of time in the block universe as having a direction, namely from earlier to later.

This leads to the main reason for caution: the A versus B debate is far more metaphysically involved and theoretical than its close connection to the metaphors make it seem. Using the moving time metaphors available in one's language is not the same as being committed to a particular metaphysical conception of time. Of course, Miller et al. are in fact suggesting that the former causes the latter. But does the latter actually obtain, 
in addition to the former? A lot of philosophical work goes into formulating both the (Atheoretic) idea that the metaphors could be getting at some literal truth about time, and the (B-theoretic) idea that time could exist without passing in this sense. To get at the subtle differences between these requires much metaphysical engineering. It's true that once one is in the business of pursuing these views (for example when doing metaphysics), A-theoretic ideas tend to have some intuitive appeal. But that's not to say that human beings in general walk around with tacit A-theoretical models in their heads.

'Is the passage of time an illusion?' has meant many things to many people.

Metaphysicians of time are one relevant group with a specific, captivating and elusive meaning in mind. When the question is understood this way, the answer is that there is no illusion of passage. $^{2}$

\section{References}

Callender, C. (2010). 'Is Time an Illusion?'. Scientific American, 302, 58-65.

doi:10.1038/scientificamerican0610-58

Eddington, A. (1920). Space, Time, and Gravitation: An Outline of the General Relativity Theory. Cambridge: Cambridge University Press.

Einstein, A. (1972). Einstein, A. \& Besso, M. 'Correspondance, 1903-1955', transl. by Pierre Speziali. Paris: Hermann.

Gruber, R., Bach, M., and Block, R. (2015). 'Perceiving Two Levels of the Flow of Time'. Journal of Consciousness Studies, 22 (5/6), 7-22.

Gruber, R., Smith, R., and Block, R. (2018). 'The Illusory Flow and Passage of Time within Consciousness: A Multidisciplinary Analysis'. Timing and Time Perception, 6, 125-153.

Ismael, J. (2010). 'Temporal Experience'. In C. Callender (ed.), Oxford Handbook on Time. Oxford University Press, 460-482. 
Ismael, J. (2012). 'Decision and the Open Future'. In The Future of the Philosophy of Time, edited by A. Bardon, Routledge, 149-169.

Hoerl, C. (2009). 'Time and Tense in Perceptual Experience'. Philosophers' Imprint, $9 / 12$.

Miller, K., Holcombe, A., and Latham, A. (forthcoming). 'Temporal Phenomenology: Phenomenological Illusion versus Cognitive Error'. Synthese.

Paul, L. (2010). ‘Temporal Experience'. Journal of Philosophy 107(7), 333-359.

Phillips, I. (2014). 'The Temporal Structure of Experience', in D. Lloyd and V. Arstila (eds.) Subjective Time: the Philosophy, Psychology, and Neuroscience of Temporality. MIT Press.

Skow, B. (2015). Objective Becoming. Oxford: Oxford University Press.

Sorkin, R. (2007). 'Relativity Theory Does not Imply that the Future Already Exists: A Counterexample', in V. Petkov (editor), Relativity and the Dimensionality of the World. Springer.

Weyl, H. (1949). Philosophy of Mathematics and Natural Science. Based on a translation by Olaf Helmer. Princeton: Princeton University Press.

${ }^{1}$ Here's what would have to be the case for our perceptions of change to involve an illusion of continuity: (i) our perceptions of change would have to be as of continuous change and persistence, so that the world would seem continuous, and (ii) that impression would have to be mistaken, for example because time would be discrete rather than continuous (or something similar).

2 This chapter was partly written while I was supported by the Yonsei University FutureLeading Research Initiative 2018 (2018-22-0100). 